\title{
Comparison of anterior and posterior trabecular bone microstructure of human mandible using cone-beam CT and micro CT
}

Norliza Ibrahim ${ }^{1 *}$, Azin Parsa², Bassam Hassan³ , Paul van der Stelt ${ }^{3}$, Rabiah A. Rahmat ${ }^{1}$, Siti M. Ismail ${ }^{1 *}$ and Irene H. A. Aartman ${ }^{3}$

\begin{abstract}
Background: The aim of this study was to compare the trabecular bone microstructures of anterior and posterior edentulous regions of human mandible using cone-beam computed tomography (CBCT) and micro computed tomography $(\mu \mathrm{CT})$.

Methods: Twenty volumes of interests consisting of six anterior and fourteen posterior edentulous regions were obtained from human mandibular cadavers. A CBCT system with a resolution of 80 um (3D Accuitomo 170, J. Morita, Kyoto, Japan) and a $\mu$ CT system with a resolution of $35 \mu \mathrm{m}$ (SkyScan 1173, Kontich, Belgium) were used to scan the mandibles. Three structural parameters namely, trabecular number (Tb.N), trabecular thickness (Tb.Th), and trabecular separation (Tb.Sp) were analysed using CTAn software (v 1.11, SkyScan, Kontich, Belgium). For each system, the measurements obtained from anterior and posterior regions were tested using independent sample t-test. Subsequently, all measurements between systems were tested using paired t-test.
\end{abstract}

Results: In CBCT, all parameters of the anterior and posterior mandible showed no significant differences ( $p>0.05)$. However, $\mu$ CT showed a significant different of Tb.Th $(p=0.023)$ between anterior and posterior region. Regardless of regions, the measurements obtained using both imaging systems were significantly different $(p \leq 0.021)$ for Tb.Th and Tb.N.

Conclusions: The current study demonstrated that only the variation of Tb.Th between anterior and posterior edentulous region of mandible can be detected using $\mu \mathrm{CT}$. In addition, CBCT is less feasible than $\mu \mathrm{CT}$ in assessing trabecular bone microstructures at both regions.

\section{Introduction}

Cone-beam computed tomography $(\mathrm{CBCT})$ is used in clinical dentistry to evaluate both the bone geometry [1] and bone density [2] mainly due to its advantages in comparison with other $3 \mathrm{D}$ imaging modalities [3]. However, the bone density assessment in CBCT images are not consistent as in medical CT [4]. Along with the

\footnotetext{
*Correspondence: norlizaibrahim@um.edu.my; sitimazlipah@ummc.edu.my ${ }^{1}$ Department of Oral and Maxillofacial Clinical Sciences, Faculty of Dentistry, University of Malaya, 50603 Kuala Lumpur, Malaysia Full list of author information is available at the end of the article
}

advancement of the CBCT scanning resolution, studies on trabecular bone microstructure using $\mathrm{CBCT}$ is becoming more available [5-9]. Furthermore, the accuracy of $\mathrm{CBCT}[5,9]$ and the influence of its scanning parameters for trabecular bone microstructure assessment have been explored [7-9].

Micro computed tomography $(\mu \mathrm{CT})$ has largely been used to analyze structural measurements of bones. Previous $\mu \mathrm{CT}$ studies showed variation in the bone microstructure measurements, depending on the site and the density of the samples [10-12]. Due to limited clinical 
applications, the assessment of trabecular microstructures for oral maxillofacial region cannot be conducted in vivo using $\mu \mathrm{CT}$ [13]. In this context the use of high resolution CBCT appears promising [3]. Therefore, it is worth to investigate the potential of $\mathrm{CBCT}$ in detecting the variations of trabecular microstructures at different bony maxillofacial regions. Human mandibular bone demonstrates a denser bone trabeculation at the anterior region in comparison to the posterior region [14]. Most bone quality studies related to dental implant are mainly limited to bone density [14] and quantity assessment [15].

Bone quality can be better assessed by measuring both bone density and trabecular microstructure parameters [13, 16-18]. Trabecular microstructure has been reported as one of the determinants to predict primary implant stability $[19,20]$, bone healing, osseointegration [21] and bone strength [22]. Thus, the aims of this study were of two-fold: (1) to compare trabecular bone microstructure parameters between anterior and posterior edentulous human mandible using $\mathrm{CBCT}$ and $\mu \mathrm{CT}$; (2) to evaluate the difference of $\mathrm{CBCT}$ and $\mu \mathrm{CT}$ in measuring trabecular bone microstructure at anterior and posterior regions of edentulous mandible.

\section{Materials and methods}

Twenty-five human mandibular cadavers were obtained from the Department of Functional Anatomy, Academic Center for Dentistry Amsterdam, and approved for research purposes. The inclusion criteria for human mandibular cadavers are edentulous posterior and/or anterior, no mandibular developmental anomaly, and no associated pathological conditions. The mandibles were scanned using a CBCT system with a resolution of $80 \mu \mathrm{m}$ (3D Accuitomo 170, J. Morita, Kyoto, Japan). The scan protocol for CBCT consisted of a $4.4 \mathrm{~cm}$ FOV using a high-resolution scan mode and a full rotation $\left(360^{\circ}\right)$. CBCT images were acquired at $90 \mathrm{kVp}, 5.0 \mathrm{~mA}$ and $17.5 \mathrm{~s}$ exposure time. After the scanning, twenty edentulous regions of the mandibles ( 6 anterior and 14 posterior) were selected to be included in this study. Subsequently, the mandibles were re-scanned using a $\mu \mathrm{CT}$ system with a resolution of $35 \mu \mathrm{m}$ (SkyScan 1173, Kontich, Belgium). During the $\mu \mathrm{CT}$ scanning, the mandibles were secured in a cylindrical styrofoam and mounted to the holder. $\mu \mathrm{CT}$ images were acquired at $130 \mathrm{kVp}$ and $61 \mathrm{~mA}$. The images from both systems were exported as Digital Imaging and Communications in Medicine (DICOM 3) files and imported into an image analysis software (Amira v4.1, Visage Imaging Inc., Carlsbad, CA).

Volume of interests (VOIs) were identified based the following criteria: the edentulous region must not be less than $5 \mathrm{~mm}$ in length and not associated with any metallic artifact. In total, twenty VOIs of the edentulous regions were segmented and compared. Then, a surface-based image registration process was performed to ensure that the CBCT's and micro-CT's VOIs were taken from the same region (Fig. 1a, b). The measurement of trabecular microstructure was obtained by importing the selected VOIs into an image structural analysis software CTAn (v 1.11, SkyScan, Kontich, Belgium) as 16-bitmap (BMP) images (65,536 Gy values). Next, to further ensure the measurements were from the same region, an additional step of matching and comparing the anatomical landmark from the VOI of CBCT (Fig. 1c) and microCT (Fig. 1d) was performed. An automated thresholding method was used to binarize the datasets to obtain the measurement of trabecular number (Tb.N), trabecular thickness (Tb.Th) and trabecular separation (Tb.Sp) (Fig. 1e, f). Throughout the analysis, the images were viewed using a 22 -inch computer monitor (full high-definition $1920 \times 1080$ pixel; Dell, Texas, United States) in a quiet and dimmed light room. All measurements were performed twice with an interval of two weeks by one trained maxillofacial radiologist with more than ten years of experience evaluating CT images.

\section{Statistical analysis}

Data were analyzed using SPSS ${ }^{\circledR}$ (v20.0, SPSS Inc., IBM Corp., Armonk, NY). Intraclass correlation coefficient (ICC) was used to assess the intraobserver's reliability. Independent sample $t$-test was performed to assess the measurement difference of each trabecular parameters (Tb.N, Tb.Th, Tb.Sp) between anterior and posterior VOIs of CBCT and $\mu$ CT respectively. Paired $t$-tests were used to assess the difference between $\mathrm{CBCT}$ and $\mu \mathrm{CT}$ measurements. The level of significance was set at $p=$ 0.05 .

\section{Results}

The ICC tests revealed excellent intraobserver reliability for all parameters and both systems $(\mathrm{CBCT} \geq 0.96$, $\mu \mathrm{CT} \geq 0.99$ ).

\section{Comparison of trabecular microstructure parameters between anterior and posterior region}

Table 1 shows the descriptive statistics and test results of the trabecular bone microstructure measurements between anterior and posterior mandibular regions using $\mathrm{CBCT}$ and $\mu \mathrm{CT}$. All measurements obtained from CBCT showed no significant differences between edentulous anterior and posterior regions parameters were not significant when analyzed using CBCT $(p \geq 0.09)$. Similarly, $\mu \mathrm{CT}$ showed no significant difference (Tb.N, $p \geq 0.580$ and Tb.Sp, $p \geq 0.381$ ) for all parameters, except for Tb.Th $(p=0.023)$. 

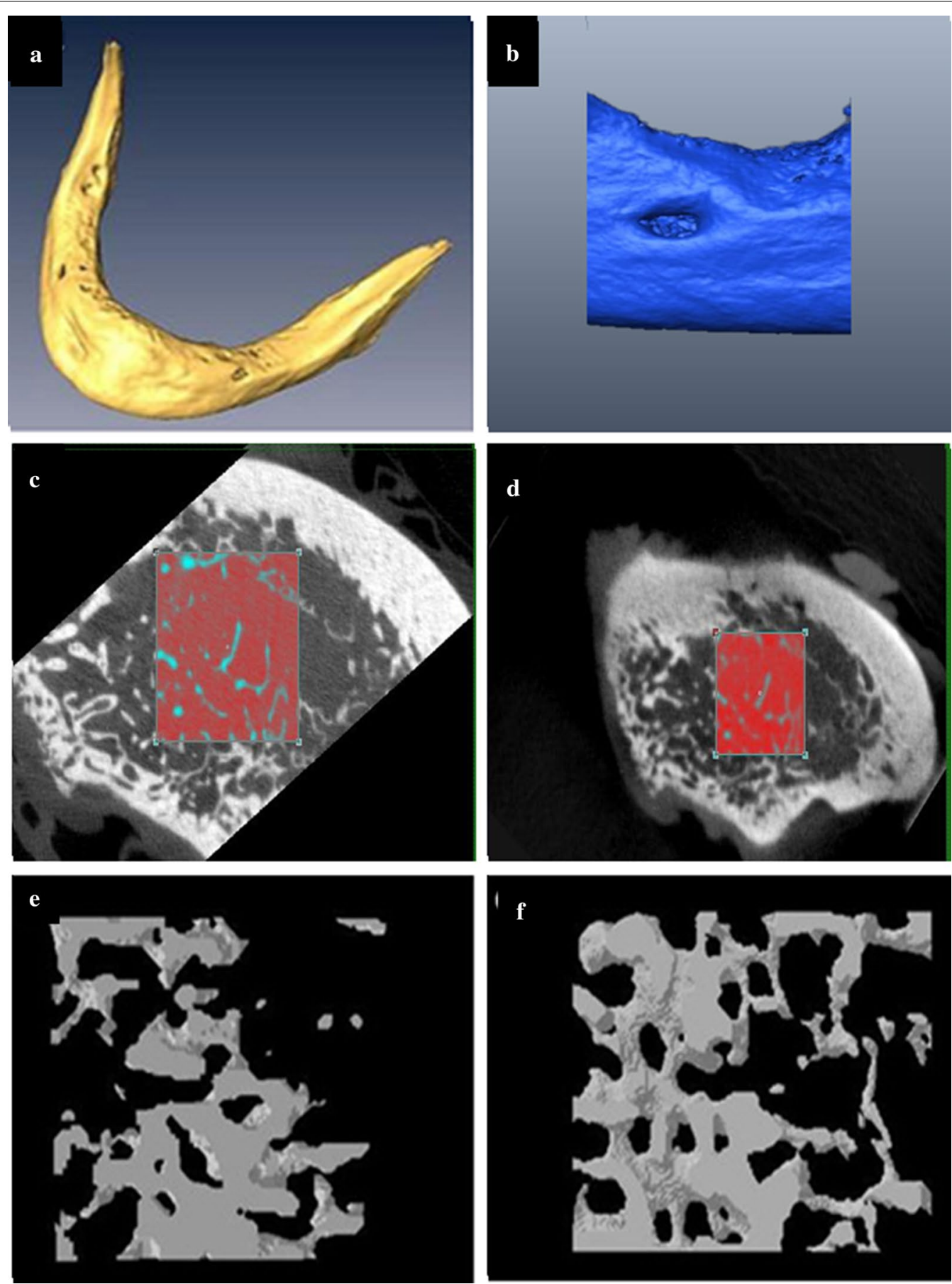

Fig. 1 Comparison of trabecular bone microstructures of human edentulous mandible using CBCT and $\mu C T$. a 3D CBCT image of an edentulous mandible. $\mathbf{b}$ 3D $\mu \mathrm{CT}$ image of a segmented edentulous mandible. Step of matching and comparing the anatomical landmark from the VOI of CBCT (c) and $\mu \mathrm{CT}(\mathbf{d})$. Trabecular bone microstructure of CBCT (e) and $\mu \mathrm{CT}$ (f) was analysed using CTAn v. 1.11 software (SkyScan, Kontich, Belgium)

\section{Comparison of trabecular microstructure parameters} between $\mathrm{CBCT}$ and $\mu \mathrm{CT}$

All parameters of trabecular bone microstructures measurements between $\mathrm{CBCT}$ and $\mu \mathrm{CT}$ were significantly different $(p \leq 0.021)$, except for Tb.Sp $(p=0.180)$ as shown in Table 2. At both regions, the Tb.N average measurement was lower in CBCT (anterior $=5.61 \mu \mathrm{m}^{-1}$, posterior $=5.60 \mu^{-1}$ ) compared to $\mu \mathrm{CT}$ (anterior $=$ 
Table 1 Inter-regional comparison of trabecular microstructure parameters using CBCT and $\mu C T$. The measurements of trabecular number (Tb.N), trabecular thickness (Tb.Th) and trabecular separation (Tb.Sp) were compared between anterior $(n=6)$ and posterior $(n=14)$ edentulous regions using the independent sample t-test.)

\begin{tabular}{|c|c|c|c|c|c|c|c|c|}
\hline Systems & Parameters & Region & Mean & SD & SEM & $\mathrm{t}$ & df & $p$ \\
\hline \multirow[t]{6}{*}{$\mathrm{CBCT}$} & Tb.N $\left(\mu \mathrm{m}^{-1}\right)$ & Anterior & 5.91 & 1.67 & 0.68 & 0.21 & 18 & 0.84 \\
\hline & & Posterior & 5.60 & 3.33 & 0.89 & & & \\
\hline & Tb.Th $(\mu \mathrm{m})$ & Anterior & 7.19 & 1.78 & 0.73 & 1.78 & 18 & 0.09 \\
\hline & & Posterior & 5.63 & 1.81 & 0.48 & & & \\
\hline & Tb.Sp $(\mu \mathrm{m})$ & Anterior & 9.47 & 2.68 & 1.10 & -0.20 & 18 & 0.85 \\
\hline & & Posterior & 9.81 & 3.71 & 0.99 & & & \\
\hline \multirow[t]{6}{*}{$\mu \mathrm{CT}$} & Tb.N $\left(\mu m^{-1}\right)$ & Anterior & 7.96 & 2.50 & 1.02 & 0.56 & 18 & 0.58 \\
\hline & & Posterior & 6.95 & 4.03 & 1.08 & & & \\
\hline & Tb.Th $(\mu \mathrm{m})$ & Anterior & 4.84 & 0.78 & 0.32 & 2.48 & 18 & $0.02^{*}$ \\
\hline & & Posterior & 3.64 & 1.07 & 0.29 & & & \\
\hline & Tb.Sp $(\mu \mathrm{m})$ & Anterior & 7.39 & 1.51 & 0.62 & -0.90 & 18 & 0.38 \\
\hline & & Posterior & 9.07 & 4.42 & 1.18 & & & \\
\hline
\end{tabular}

*Significant difference was accepted at $p<0.05$

Table 2 Intra-regional comparison of trabecular microstructure parameters using CBCT and $\mu C T$. The measurements of trabecular number (Tb.N), trabecular thickness (Tb.Th) and trabecular separation (Tb.Sp) of either anterior $(n=6)$ or posterior $(n=14)$ regions were compared using the independent sample t-test

\begin{tabular}{|c|c|c|c|c|c|c|c|c|c|c|}
\hline \multirow[t]{2}{*}{ Parameters } & \multirow[t]{2}{*}{ Regions } & \multicolumn{3}{|l|}{ CBCT } & \multicolumn{3}{|l|}{$\mu \mathrm{CT}$} & \multicolumn{3}{|l|}{$t$-test } \\
\hline & & Mean & SD & SEM & Mean & SD & SEM & $t$ & df & $p$ \\
\hline \multirow[t]{2}{*}{ Tb.N $\left(\mu \mathrm{M}^{-1}\right)$} & Anterior & 5.61 & 1.67 & 0.68 & 7.96 & 2.50 & 1.02 & 3.46 & 5 & $0.018^{*}$ \\
\hline & Posterior & 5.60 & 3.33 & 0.89 & 6.95 & 4.03 & 1.08 & 4.21 & 13 & $0.001 *$ \\
\hline \multirow[t]{2}{*}{ Tb.Th $(\mu \mathrm{M})$} & Anterior & 7.19 & 1.78 & 0.73 & 4.84 & 0.78 & 0.32 & -4.73 & 5 & $0.005^{*}$ \\
\hline & Posterior & 5.63 & 1.81 & 0.48 & 3.64 & 1.07 & 0.29 & -4.90 & 13 & $0.001^{*}$ \\
\hline \multirow[t]{2}{*}{ Tb.Sp ( $\mu \mathrm{M})$} & Anterior & 9.47 & 2.68 & 1.10 & 7.39 & 1.51 & 0.62 & -3.33 & 5 & $0.021^{*}$ \\
\hline & Posterior & 9.81 & 3.71 & 0.99 & 9.07 & 4.42 & 1.18 & -1.42 & 13 & 0.180 \\
\hline
\end{tabular}

Tb.N trabecular number, Tb.Th trabecular thickness, Tb.Sp trabecular spacing, Ant anterior, Post posterior, SD standard deviation, SEM standard error of mean

*The difference is significant at $p<0.05$. Significant difference was accepted at $p<0.05$

$7.96 \mu \mathrm{m}^{-1}$, posterior $=6.95 \mu \mathrm{m}^{-1}$. In contrast, Tb.Sp and Tb.Th were higher in CBCT ( Tb.Sp anterior $=9.47 \mu \mathrm{m}$, Tb.Sp posterior $=9.81 \mu \mathrm{m}$; Tb.Th anterior $=7.19 \mu \mathrm{m}$, Tb.Th posterior $=5.63 \mu \mathrm{m})$ than $\mu \mathrm{CT}(\mathrm{Tb} . \mathrm{Sp}$ anterior $=7.39 \mu \mathrm{m}$, Tb.Sp posterior $=9.07 \mu \mathrm{m}$; Tb.Th anterior $=$ $4.84 \mu \mathrm{m}$, Tb.Th posterior $=3.64 \mu \mathrm{m}$ ).

\section{Discussion}

Trabecular microstructure is one of the important determinants for bone quality. The latest CBCT generation offers a high scanning resolution which is adequate for trabecular microstructural evaluation [3, 4, 6, 23]. Prior to its application for clinical evaluation, the accuracy of CBCT measurements have been compared to a reference modality i.e. $\mu \mathrm{CT}[3,5,6,9]$. However, most CBCT based studies are constrained to the technical influence of various scanning parameters [6-8]. Assessment of the regional bone quality variations is important in predicting the success of implant treatment at different sites of human mandible [14, 15]. Kim et al. [24] demonstrated the microstructural differences between various regions of maxilla and mandible. However, the study was limited to $\mu \mathrm{CT}$ and dentate regions. To our knowledge, this is the first study assessing microstructural bone parameters between anterior and posterior edentulous regions of mandible using CBCT.

Trabecular bone varies according to the mandibular regions [24-26]. This is due to the disparity of complex trabecular configurations $[17,27]$. Unlike $\mu C T$, this current study demonstrated that $\mathrm{Tb}$.Th measurement was not significantly different in CBCT. However, it was the only the potential parameter to distinguish both regions (Table 1). In contrast, other $\mu \mathrm{CT}$ and histomicromorphic studies $[17,27]$ have found significant differences in more than one parameter. This might be due the differences in 
bone density [10, 16, 17], type of specimens [17], scanning protocols [7] and the system's technology [28] used in this study.

Microstructural evaluation is highly dependent on the image resolution [29-31]. The current study demonstrated that structural measurements obtained from both CBCT $(80 \mu \mathrm{m})$ and $\mu \mathrm{CT}(35 \mu \mathrm{m})$ were different, except for Tb.Sp at posterior region (Table 2). This result was concordance to a $\mu \mathrm{CT}$ study reported by Fanuscu and Chang [17] that used different resolution $(12-110 \mu \mathrm{m})$. Although a small voxel size $(<100 \mu \mathrm{m})$ is recommended for microstructural evaluation $[10,17,31]$, the resolution for an accurate analysis is still dependent on the bone origin [11,32] and regional density [17]. It was described that a low-density bone may exhibit a wide variation of structural measurements in regards to the thresholding technique imposed [16]. Thus, the trabecular structural parameters at different density regions might be over- or under-estimated resulting in unfavorable differences in this study.

Since this study was the first of its kind, the optimum resolution for CBCT microstructural assessment was not available in the literature. Therefore, a specific resolution should be set when comparing different types of bone density in future studies. This study has only assessed the difference of trabecular bone microstructure at two different regions of edentulous mandibles. Hence, further studies should be conducted to assess maxillary regions with different bone density, scanning protocols and the system's technology.

\section{Conclusions}

The current study has suggested that micro CT can depict the differences of Tb.Th between the anterior and posterior edentulous regions of mandible. The use of CBCT is less feasible due to inadequate resolution in depicting structural differences at different regions.

\section{Acknowledgements}

The authors would like to thank Department of Oral and Maxillofacial Radiology, Academic Center for Dentistry Amsterdam (ACTA).

\section{Authors' contributions}

$\mathrm{NI}$ handled project administration, methodology, data analysis, visualization, the main manuscript writing and preparation. AP curated data and sought resources. $\mathrm{BH}$ and $\mathrm{P}$ van der $\mathrm{S}$ supervised the research and reviewed the manuscript. SMI and RA-AR reviewed and revised the manuscript. IHAA performed statistical analysis and interpretation, and revised the manuscript. All authors read and approved the final manuscript.

\section{Funding}

Not applicable.

\section{Availability of data and materials}

The datasets generated during and analysed during the current study are not publicly available because it contains personal information but are available from the first author on reasonable request.

\section{Declarations}

Ethics approval and consent to participate

The human mandibular cadavers use in this study were obtained from the Department of Functional Anatomy, Academic Center for Dentistry, Amsterdam (ACTA). All cadavers were donated for research purposes following written informed consent from the individual or relatives. The handling of the cadaveric mandibles in this study was conducted according to the ethical guidelines of the ACTA. Thus, no informed consent was required to use the mandibular cadavers.

\section{Consent for publication}

Not applicable.

\section{Competing interests}

The authors declare that they have no conflicts of interest.

\section{Author details}

${ }^{1}$ Department of Oral and Maxillofacial Clinical Sciences, Faculty of Dentistry, University of Malaya, 50603 Kuala Lumpur, Malaysia. ${ }^{2}$ Department of Diagnostic Sciences, School of Dentistry, The University of North Carolina, Chapel Hill, USA. ${ }^{3}$ Department of Oral and Maxillofacial Radiology, Academic Center for Dentistry Amsterdam (ACTA), Louwesweg 1, 1066 EA Amsterdam, The Netherlands.

Received: 20 November 2020 Accepted: 12 April 2021

Published online: 08 May 2021

\section{References}

1. Zhang X, Li Y, Ge Z, Zhao H, Miao L, Pan Y. The dimension and morphology of alveolar bone at maxillary anterior teeth in periodontitis: a retrospective analysis-using CBCT. Int J Oral Sci. 2020;12(1):4.

2. Soardi CM, Suárez-López del Amo F, Galindo-Moreno P, Catena A, Zaffe D, Wang HL. Reliability of cone beam computed tomography in determining mineralized tissue in augmented sinuses. Int J Oral Maxillofac Implants. 2016;31(2):352-8.

3. Van Dessel J, Nicolielo LF, Huang Y, Coudyzer W, Salmon B, Lambrichts I, Jacobs R. Accuracy and reliability of different cone beam computed tomography (CBCT) devices for structural analysis of alveolar bone in comparison with multislice CT and micro-CT. Eur J Oral Implantol. 2017;10(1):95-105.

4. Pauwels R, Jacobs R, Singer SR, Mupparapu M. CBCT-based bone quality assessment: are Hounsfield units applicable? Dentomaxillofac Radiol. 2015:44(1):20140238.

5. Kulah K, Gulsahi A, Kamburoğlu K, Geneci F, Ocak M, Celik HH, Ozen T. Evaluation of maxillary trabecular microstructure as an indicator of implant stability by using 2 cone beam computed tomography systems and micro-computed tomography. Oral Surg Oral Med Oral Pathol Oral Radiol. 2019;127(3):247-56.

6. Van Dessel J, Huang Y, Depypere M, Rubira-Bullen I, Maes F, Jacobs R. A comparative evaluation of cone beam CT and micro-CT on trabecular bone structures in the human mandible. Dentomaxillofac Radiol. 2013;42(8):20130145.

7. Pauwels R, Faruangsaeng T, Charoenkarn T, Ngonphloy N, Panmekiate S. Effect of exposure parameters and voxel size on bone structure analysis in CBCT. Dentomaxillofac Radiol. 2015;44(8):20150078.

8. Ibrahim N, Parsa A, Hassan B, van der Stelt P, Aartman IH, Nambiar P. Influence of object location in different FOVs on trabecular bone microstructure measurements of human mandible: a cone beam CT study. Dentomaxillofac Radiol. 2014;43(2):20130329.

9. Van Dessel J, Nicolielo LF, Huang Y, Slagmolen P, Politis C, Lambrichts I, et al. Quantification of bone quality using different cone beam computed tomography devices: accuracy assessment for edentulous human mandibles. Eur J Oral Implantol. 2016;9(4):411-24.

10. Isaksson H, Töyräs J, Hakulinen M, Aula A, Tamminen I, Julkunen P, et al. Structural parameters of normal and osteoporotic human trabecular bone are affected differently by microCT image resolution. Osteoporos Int. 2011;22(1):167-77. 
11. Bouxsein ML, Boyd SK, Christiansen BA, Guldberg RE, Jepsen KJ, Müller R. Guidelines for assessment of bone microstructure in rodents using micro-computed tomography. J Bone Miner Res. 2010;25(7):1468-86.

12. Irie MS, Rabelo GD, Spin-Neto R, Dechichi P, Borges JS, Soares PBF. Use of micro-computed tomography for bone evaluation in dentistry. Braz Dent J. 2018;29(3):227-38.

13. de Oliveira RC, Leles CR, Lindh C, Ribeiro-Rotta RF. Bone tissue microarchitectural characteristics at dental implant sites. Part 1: identification of clinical-related parameters. Clin Oral Implants Res. 2012;23(8):981-6.

14. Fuh LJ, Huang HL, Chen CS, Fu KL, Shen YW, Tu MG, Shen WC, Hsu JT. Variations in bone density at dental implant sites in different regions of the jawbone. J Oral Rehabil. 2010;37(5):346-51.

15. Ko YC, Huang HL, Shen YW, Cai JY, Fuh LJ, Hsu JT. Variations in crestal cortical bone thickness at dental implant sites in different regions of the jawbone. Clin Implant Dent Relat Res. 2017;19(3):440-6.

16. Wirth AJ, Goldhahn J, Flaig C, Arbenz P, Müller R, van Lenthe GH. Implant stability is affected by local bone microstructural quality. Bone. 2011;49(3):473-8.

17. Fanuscu Ml, Chang TL. Three-dimensional morphometric analysis of human cadaver bone: microstructural data from maxilla and mandible. Clin Oral Implants Res. 2004;15(2):213-8.

18. Kim YS, Han JJ, Lee J, Choi HS, Kim JH, Lee T. The correlation between bone mineral density/trabecular bone score and body mass index, height, and weight. Osteoporos Sarcopenia. 2017;3(2):98-103.

19. Hsu JT, Huang HL, Chang CH, Tsai MT, Hung WC, Fuh LJ. Relationship of three-dimensional bone-to-implant contact to primary implant stability and peri-implant bone strain in immediate loading: microcomputed tomographic and in vitro analyses. Int J Oral Maxillofac Implants. 2013;28(2):367-74.

20. Kang SR, Bok SC, Choi SC, Lee SS, Heo MS, Huh KH, et al. The relationship between dental implant stability and trabecular bone structure using cone-beam computed tomography. J Periodontal Implant Sci. 2016:46(2):116-27.

21. Minkin C, Marinho VC. Role of the osteoclast at the bone-implant interface. Adv Dent Res. 1999;13(1):49-56.

22. Majumdar S, Lin J, Link T, Millard J, Augat P, Ouyang X, et al. Fractal analy sis of radiographs: assessment of trabecular bone structure and prediction of elastic modulus and strength. Med Phys. 1999;26(7):1330-40.
23. Liang X, Zhang Z, Gu J, Wang Z, Vandenberghe B, Jacobs R, et al. Comparison of micro-CT and cone beam CT on the feasibility of assessing trabecular structures in mandibular condyle. Dentomaxillofac Radiol. 2017;46(5):20160435

24. Kim JE, Shin JM, Oh SO, Yi WJ, Heo MS, Lee SS, et al. The three-dimensional microstructure of trabecular bone: analysis of site-specific variation in the human jaw bone. Imaging Sci Dent. 2013;43(4):227-33.

25. von Wowern N. Variations in structure within the trabecular bone of the mandible. Scand J Dent Res. 1977:85(7):613-22.

26. Lee JH, Kim HJ, Yun JH. Three-dimensional microstructure of human alveolar trabecular bone: a micro-computed tomography study. J Periodontal Implant Sci. 2017;47(1):20-9.

27. Ulm C, Tepper G, Blahout R, Rausch-Fan X, Hienz S, Matejka M. Characteristic features of trabecular bone in edentulous mandibles. Clin Oral Implants Res. 2009;20(6):594-600.

28. Schulze R, Heil U, Gross D, Bruellmann DD, Dranischnikow E, Schwanecke $U$, Schoemer E. Artefacts in CBCT: a review. Dentomaxillofac Radiol. 2011;40(5):265-73.

29. Kothari M, Keaveny TM, Lin JC, Newitt DC, Genant HK, Majumdar S. Impact of spatial resolution on the prediction of trabecular architecture parameters. Bone. 1998;22(5):437-43.

30. Müller R, Koller B, Hildebrand T, Laib A, Gianolini S, Rüegsegger P. Resolution dependency of microstructural properties of cancellous bone based on three-dimensional mu-tomography. Technol Health Care. 1996:4(1):113-9.

31. Christiansen BA. Effect of micro-computed tomography voxel size and segmentation method on trabecular bone microstructure measures in mice. Bone Rep. 2016;5:136-40.

32. Sode M, Burghardt AJ, Nissenson RA, Majumdar S. Resolution dependence of the non-metric trabecular structure indices. Bone. 2008:42(4):728-36.

\section{Publisher's note}

Springer Nature remains neutral with regard to jurisdictional claims in published maps and institutional affiliations.
Ready to submit your research? Choose BMC and benefit from:

- fast, convenient online submission

- thorough peer review by experienced researchers in your field

- rapid publication on acceptance

- support for research data, including large and complex data types

- gold Open Access which fosters wider collaboration and increased citations

- maximum visibility for your research: over $100 \mathrm{M}$ website views per year

At BMC, research is always in progress.

Learn more biomedcentral.com/submissions 